\title{
Sex and age interact to determine clinicopathologic differences in Alzheimer's disease
}

\author{
Amanda M. Liesinger ${ }^{1} \cdot$ Neill R. Graff-Radford ${ }^{2} \cdot$ Ranjan Duara $^{4} \cdot$ Rickey E. Carter ${ }^{3} \cdot$ Fadi S. Hanna Al-Shaikh ${ }^{1}$. \\ Shunsuke Koga ${ }^{1} \cdot$ Kelly M. Hinkle $^{1} \cdot$ Sarah K. DiLello ${ }^{1} \cdot$ McKenna F. Johnson $^{1} \cdot$ Adel Aziz $^{2}$ - Nilufer Ertekin-Taner ${ }^{1,2}$. \\ Owen A. Ross ${ }^{1} \cdot$ Dennis W. Dickson ${ }^{1} \cdot$ Melissa E. Murray ${ }^{1}$ (i)
}

Received: 18 May 2018 / Revised: 8 September 2018 / Accepted: 9 September 2018 / Published online: 15 September 2018

(c) The Author(s) 2018

\begin{abstract}
Women reportedly make up two-thirds of Alzheimer's disease (AD) dementia sufferers. Many estimates regarding AD, however, are based on clinical series lacking autopsy confirmation. The Florida Autopsied Multi-Ethnic (FLAME) cohort was queried for $\mathrm{AD}$ cases with a total of 1625 identified ranging in age from 53 to 102 years at death. Standard neuropathologic procedures were employed and clinical information was retrospectively collected. Clinicopathologic and genetic data (MAPT and $A P O E$ ) were stratified by sex. Within the neuropathologically diagnosed AD cohort, the overall number of women and men did not differ. Men were younger at onset of cognitive symptoms, had a shorter disease duration, and more often had atypical (non-amnestic) clinical presentations. The frequency of autopsy-confirmed AD among women and men stratified by age at death revealed an inverse U-shaped curve in men and a U-shaped curve in women, with both curves having inflections at approximately 70 years of age. Regional counts of neurofibrillary tangles differed in women and men, especially when examined by age intervals. Women had overall greater severity of neurofibrillary tangle counts compared to men, especially in the hippocampus. Men were more often classified as hippocampal sparing $\mathrm{AD}$, whereas limbic predominant $\mathrm{AD}$ was more common in women. Men and women did not differ in frequency of MAPT haplotype or APOE genotype. Atypical clinical presentations, younger age at onset and shorter disease duration were more frequent in men, suggesting that the lower reported frequency of $\mathrm{AD}$ in men may be due to more frequent atypical clinical presentations not recognized as $\mathrm{AD}$. Our data suggest that neuropathologically diagnosed AD cases have the same frequency of women and men, but their clinical presentations and ages at onset tend to differ.
\end{abstract}

Keywords Alzheimer's disease $\cdot$ Neuropathology $\cdot$ Sex $\cdot$ Gender $\cdot$ Neurofibrillary tangle $\cdot$ Atypical $\cdot$ Plaques $\cdot$ Age $\cdot$ Young onset $\cdot$ Late onset $\cdot$ Autopsy $\cdot$ Postmortem

Electronic supplementary material The online version of this article (https://doi.org/10.1007/s00401-018-1908-x) contains supplementary material, which is available to authorized users.

Melissa E. Murray

murray.melissa@mayo.edu

1 Departments of Neuroscience, Mayo Clinic, Jacksonville, FL 32224, USA

2 Departments of and Neurology, Mayo Clinic, Jacksonville, FL, USA

3 Biomedical Statistics and Informatics, Mayo Clinic, Jacksonville, FL, USA

4 Wien Center for Alzheimer's Disease and Memory Disorders, Mount Sinai Medical Center, Miami Beach, FL, USA

\section{Introduction}

The strongest risk factor for Alzheimer's disease (AD) is increasing age [38]. The impact of sex differences on AD risk between women and men, however, has been variably interpreted [3, 20, 28, 31, 37]. Evidence from multi-site studies suggests that women make up nearly two-thirds of patients with AD dementia in North America [28]. The prevalence of $\mathrm{AD}$ dementia among women and men has also been increasing steadily in European ethnic groups, particularly for women after age 90 [31, 37].

Postmortem studies have revealed that women tend to have greater $\mathrm{AD}$ pathology $[5,13]$, but men more often present with atypical clinical syndromes (e.g., frontotemporal dementia, corticobasal syndrome) [14, 24]. We developed 
a mathematical algorithm based upon density and distribution of neurofibrillary tangle counts to classify three neuropathologic subtypes of $\mathrm{AD}$ : hippocampal sparing $\mathrm{AD}$, typical AD, and limbic predominant AD [24]. Further investigation revealed that $63 \%$ of the hippocampal sparing $\mathrm{AD}$ were men, while $69 \%$ of limbic predominant $\mathrm{AD}$ were women. Moreover, the hippocampal sparing AD subtype had an average age at onset of cognitive symptoms at 63 years and shorter average disease duration of 8 years. In contrast, limbic predominant $\mathrm{AD}$ had an older average age at onset of 76 years old and longer disease duration of 10 years. We found that nearly $50 \%$ of hippocampal sparing AD had an atypical (non-amnestic) clinical syndrome, whereas more than $90 \%$ of limbic predominant AD had an AD dementia clinical presentation.

We hypothesized that atypical clinical presentations may lead to more frequent clinical misdiagnosis of $\mathrm{AD}$ in men, and thereby result in an apparent reduced frequency of $\mathrm{AD}$ in men. Thus, we proposed to investigate differences in the frequency of $\mathrm{AD}$ utilizing an autopsy-confirmed series from the Florida Autopsied Multi-Ethnic (FLAME) cohort. The objectives of this study were: (1) to examine differences between women and men, with respect to demographics, clinical course, genetics, and neuropathologic measures; and (2) to examine sex differences in neuroanatomic distributions of amyloid- $\beta$ plaques and neurofibrillary tangles stratified by age.

\section{Materials and methods}

\section{Study samples}

The FLAME cohort is based on a collection 2809 autopsied brains from the State of Florida Brain Bank [4], which is housed at Mayo Clinic in Jacksonville, Florida. The State of Florida Brain Bank is a component of the Alzheimer's Disease Initiative supported in part by the State of Florida Department of Elder Affairs (http://elderaffairs.state.fl.us/ doea/alz.php). From the $n=2809$ available brains accessioned prior to August 2015, $n=1625$ were neuropathologically diagnosed $\mathrm{AD}$ cases without known $\mathrm{AD}$ mutations [suppl. Fig. 1 (Online Resource 1)]. Further details on the cases are provided in the "Results" section. The autopsyconfirmed AD cases from the FLAME cohort are derived from a consecutive series of patients referred via memory disorder clinics distributed throughout Florida, community-based education seminars for caregivers of dementia patients, and Alzheimer's Association educational support groups. It is important to note that clinical diagnosis was not used to include or exclude neuropathologically diagnosed AD cases. All research was done on postmortem samples that are regarded by the Mayo Clinic Institutional Review
Board as exempt from the requirements of research on human subjects. All brains were acquired with appropriate ethical approval, and the study was approved by the Mayo Clinic Institutional Review Board (IRB 16-003061).

\section{Clinicopathologic assessment}

Antemortem clinical history was retrospectively abstracted from available medical records, which included a brain bank questionnaire, medical history, neurologic examinations, and/or neurology or medical follow-up notes. Demographic information was recorded on the following with availability noted in parentheses: self-reported ethnoracial status [1625/1625 (100\%)], family history of cognitive impairment [1576/1625 (97\%)], years of education [908/1625 (56\%)], and age at death $[1625 / 1625(100 \%)]$. Age at onset of cognitive symptoms was abstracted by specifically reviewing the clinical history for evidence of cognitive impairment. Other neurologic presentations (e.g., parkinsonism, spasticity, muscle weakness) were not documented for age at onset. Cognitive complaints could include, but were not limited to issues with memory (e.g., repetitive conversation, spatial disorientation), problems with naming, word finding difficulty, difficulty with math, visuospatial skills, manipulation of objects, and/or behavioral changes [34]. The date on which cognitive symptoms were first noted was recorded and subtracted from date of birth to obtain age at onset in years, which was available for 1148/1625 (71\%). The date of symptom onset was subtracted from date of death to obtain disease duration of cognitive symptoms in years, which was available for 1148/1625 (71\%). All available Mini-Mental Status Examination (MMSE) dates and accompanying test scores (0-30 points) were recorded. There were $723 / 1625$ (44\%) cases with at least one MMSE test date and 465/1625 (29\%) with two or more MMSE test dates. All MMSE test dates were subtracted from date of death to obtain a test interval before death. The final MMSE test score was assessed for those individuals who were tested within 3 years of death. Rate of cognitive decline was calculated as the reduction of MMSE points per year, using three or more MMSE test dates relative to date of death. The rate was calculated as the slope of the linear regression line through the MMSE test intervals (dependent variable) and MMSE test scores (independent variable). Clinical diagnosis was classified as non-AD or atypical if the patient was given an antemortem diagnosis of corticobasal syndrome, primary progressive aphasia, posterior cortical atrophy, frontotemporal dementia, dementia with Lewy bodies, or any of several less common diagnoses (e.g., Creutzfeldt-Jakob disease, dementia of unknown etiology, normal pressure hydrocephalus, Parkinson's disease, Parkinson's disease dementia, progressive supranuclear palsy, and vascular dementia). 
Systematic and standardized neuropathologic examinations and procedures were performed as previously described [24]. Briefly, neuropathologic insults were quantified using thioflavin-S fluorescent microscopy to count neurofibrillary tangles and senile plaques in association cortices (frontal, temporal, and parietal), primary cortices (visual and motor), hippocampus (CA1 and subiculum), and amygdala; as well as the presence of these senile plaques in basal ganglia and cerebellum. Using an Olympus $\mathrm{BH} 2$ fluorescence microscope (Center Valley, PA, USA), slides were examined at low magnification to identify areas of greatest lesion density for plaques and tangles separately. All layers of cortex were reviewed with greatest density of plaques typically observed in the middle or upper layers and greatest density of tangles typically observed in layer III or layer V pyramidal layers. The pyramidal layer of the hippocampus is used to count plaques and tangles. Two or more microscopic fields are counted to find area of greatest density based upon visual assessment of lesion burden at low magnification. Senile plaques were counted using a $10 \times$ objective $\left(3 \mathrm{~mm}^{2}\right.$ microscopic field) with 50 used as maximum, based on twice the density recommended to meet Khachaturian criteria for Alzheimer's disease [17]. Neurofibrillary tangles were counted using a $40 \times$ objective $\left(0.125 \mathrm{~mm}^{2}\right.$ microscopic field $)$ with no maximum. The data were analyzed and presented as counts per microscopic field. This information was then used to assign a Thal amyloid phase [36], Braak tangle stage [9], and AD subtype [24, 25]. Doublestaining of thioflavin-S and immunohistochemical markers was performed as previously described [18], but further details are provided in the suppl. Methods (Online Resource 2). Figures 1 and 2 provide representative images to illustrate overlap between thioflavin-S and immunofluorescent labeling with a primary antibody to amyloid- $\beta$ or tau, respectively. Validation of our thioflavin-S neurofibrillary tangle findings was performed using PHF-1 (1:1000, mouse, anti-phospho-serine 396/404 tau, gift from Peter Davies). The presence of Lewy body disease was assessed using the antibody NACP (1:3000, rabbit, amino acids $98-115$ with a cysteine residue at its C-terminus [7]). Lewy body disease subtypes were assessed based on neuroanatomic distribution of Lewy bodies [22]. TAR DNA binding protein 43 (TDP-43, rabbit, amino acids 220-227 in the 25-kDa C-terminal fragment [39]) pathology was assessed in the hippocampus for positivity [1]. Cerebrovascular disease was assessed based on methods used in the study by Jellinger and Attems (2003) [16]. Standard methods for genetic screening of $A P O E$ and $M A P T$ were used, as previously described $[24,25]$.

To validate thioflavin-S findings, the hippocampus (CA1 and subiculum) and association cortices (frontal, temporal, and parietal) in 135 autopsy-confirmed AD cases were examined. PHF-1 slides were digitally scanned and analyzed using Aperio technology (Leica Biosystems, Buffalo
Grove, IL), as previously described [24]. Briefly, the pyramidal layer of CA1 and subiculum were traced. The interface between the radiatum layer and molecular layer was used to define the superior border, while the alveus was used to define the inferior border. The association cortices were traced along the strait of the gyrus. A custom-designed color deconvolution macro was designed to recognize the strong intensity of neurofibrillary tangles to reduce quantification of the neuritic pathology. The quantitative data are summarized as a percent burden, which represents the percent of immunopositive labeling per $\mathrm{mm}^{2}$.

\section{Statistical analysis}

SigmaPlot v12 was used to perform all statistical analyses. Significance was set at $p$ value $<0.05$. We assessed two-sample analyses using the Wilcoxon rank sum test, reporting the median and interquartile ranges. We assessed categorical variables using Chi square analyses, reporting number of cases (over appropriate denominator) and percent in category. Spearman Rank Order Correlation was performed to examine relationship between thioflavin-S and PHF-1 immunohistochemistry. Regression modeling of postmortem findings was performed to examine potential contribution of independent clinical parameters (age at onset, disease duration, education). Each neuropathologic variable was separately assessed as the independent variable using multiple linear regression for continuous variables and multiple logistic regression models for discrete variables.

\section{Role of the funding source}

The sponsors of this study had no role in study design, collection of data, analysis of data, interpretation of data, or writing of the report. The corresponding author had full access to all the data in the study and had final responsibility for the decision to submit for publication.

\section{Results}

\section{Stratification of men and women by clinical diagnostic group}

Out of the 2809 autopsied FLAME study cases, regardless of clinical or neuropathologic diagnosis, there were $n=1435$ (51\%) men with an age range of 36-99 and $n=1374$ (49\%) women with an age range of 36-104. Within this cohort, $n=1625 / 2809$ (58\%) were neuropathologically diagnosed as $\mathrm{AD}$ with an age range of 53-102. Of the autopsy-confirmed $\mathrm{AD}$, adequate clinical information was available on $n=1356 / 1625$ cases $(83 \%)$. These AD cases were stratified into three clinical diagnostic groups: (1) AD dementia, 


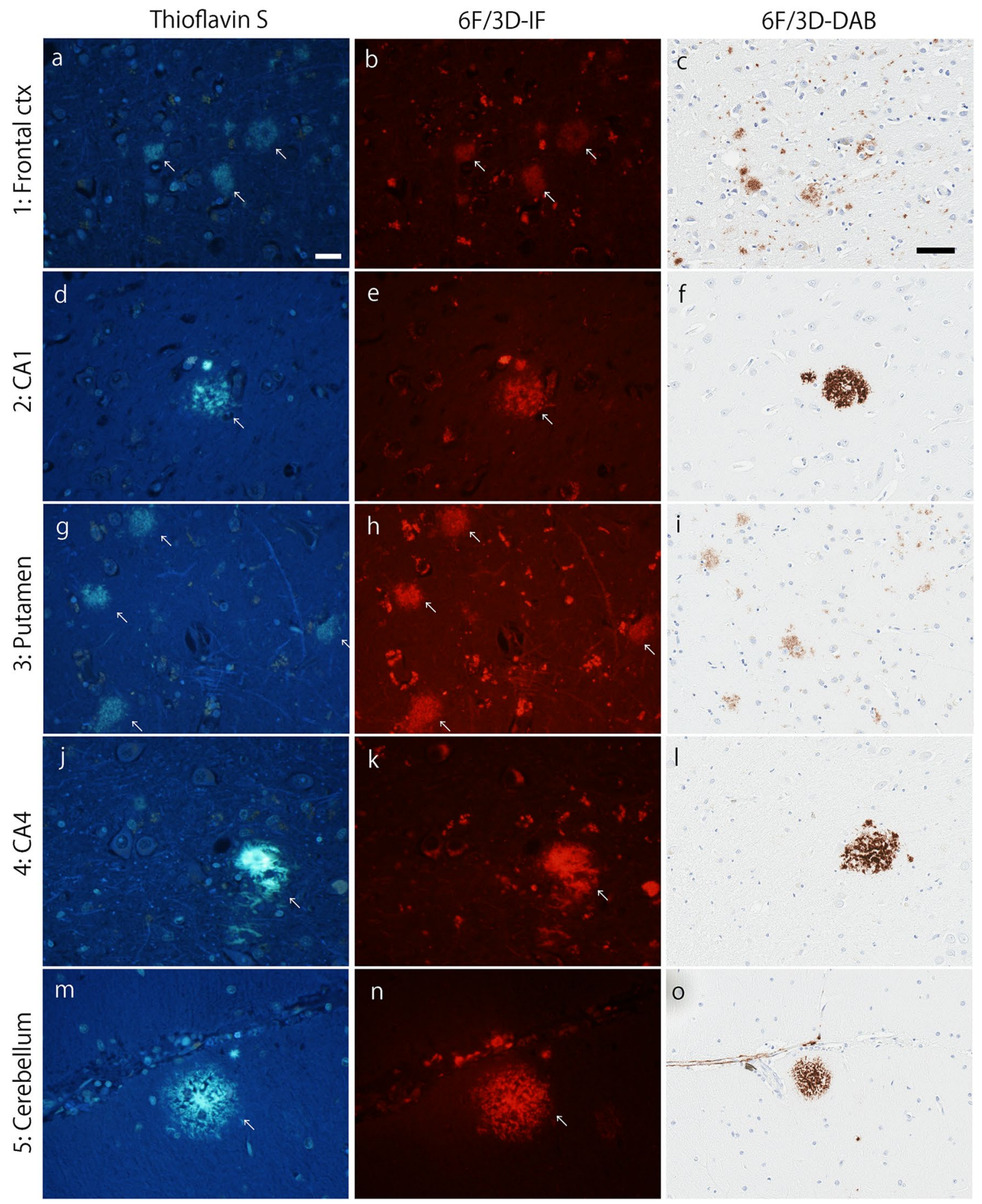

Fig. 1 Thal amyloid phase. Representative images of thioflavin-S fluorescence (left column), immunofluorescent labeling of amyloid- $\beta$ (6F/3D; middle column), and immunohistochemical 3,3'-diaminobenzidine (DAB) staining of $6 \mathrm{~F} / 3 \mathrm{D}$ (right column) in each Thal amyloid phase. Sections from the frontal cortex (phase $1 ; \mathbf{a}-\mathbf{c}$ ), the pyramidal

(2) AD dementia with a differential of an atypical clinical syndrome, or (3) atypical clinical syndrome [suppl. Fig. 1 (Online Resource 1), Table 1]. Among those who presented clinically with an AD dementia syndrome, $58 \%$ were women layer of the CA1 subsector of the hippocampus (phase 2; d-f), putamen (phase $3 ; \mathbf{g}-\mathbf{i}$ ), CA4 subsector of the hippocampus (phase $\mathbf{j}-\mathbf{l}$ ), and the molecular layer of the cerebellum (phase $\mathbf{m}-\mathbf{o}$ ) are shown. Arrows indicate amyloid- $\beta$ plaques that are labeled with both thioflavin-S and $6 \mathrm{~F} / 3 \mathrm{D}$. Bars $=20 \mu \mathrm{m}$

and $42 \%$ were men $(p<0.001)$; whereas, among those who presented with an atypical clinical syndrome, $38 \%$ were women and $62 \%$ were men $(p<0.001)$. In addition, cases presenting with an atypical syndrome were younger at 


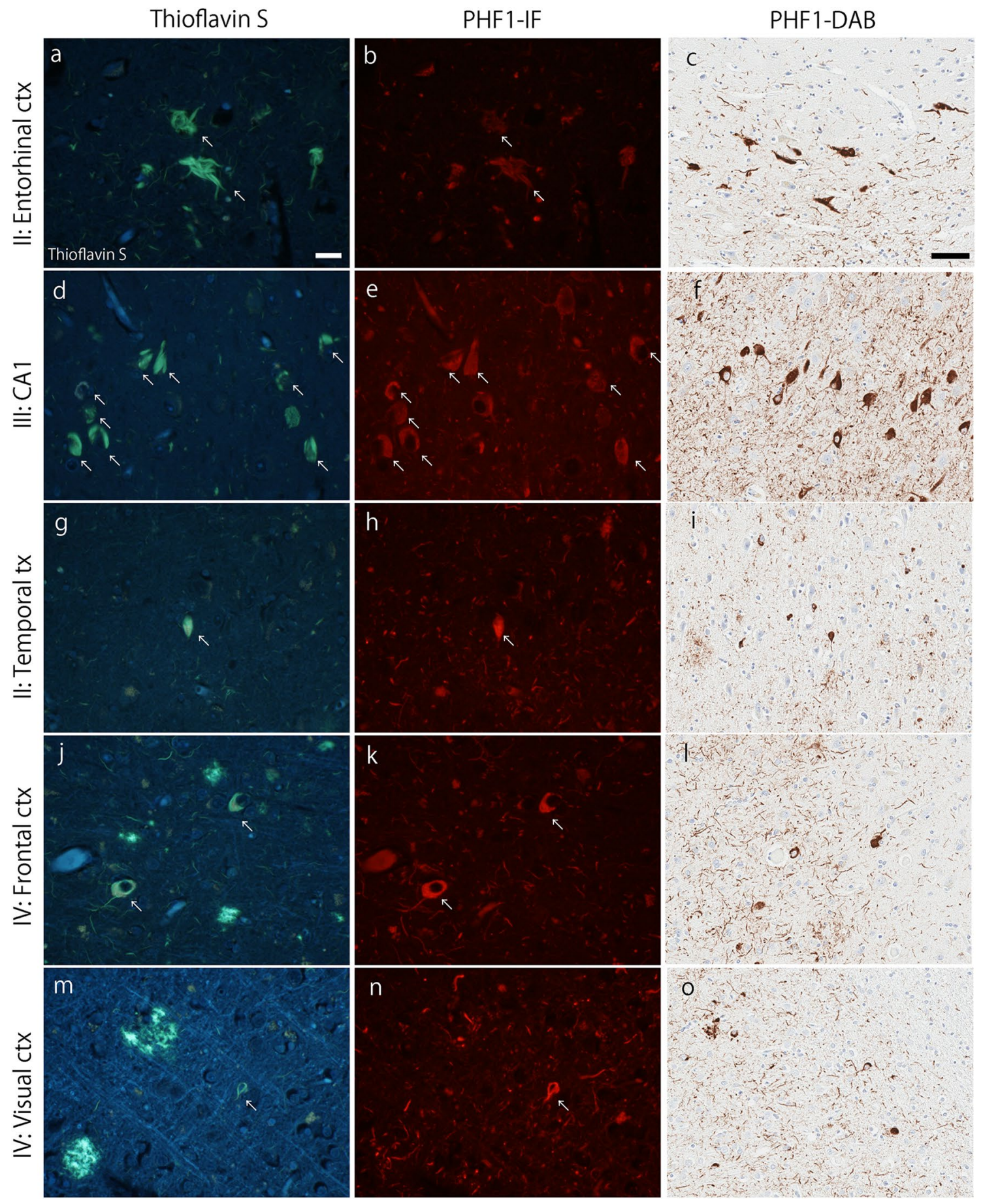

Fig. 2 Braak tangle stage. Representative images of thioflavin$\mathrm{S}$ fluorescence (left column), immunofluorescent labeling of tau (PHF-1; middle column), and immunohistochemical 3,3'-diaminobenzidine (DAB) staining of PHF-1 (right column) in each Braak tangle stage. Sections from the entorhinal cortex (stage II; a-c), the

age at onset ( 70 years) compared to those presenting with AD dementia (73 years) $(p<0.001)$. The cases presenting with atypical clinical syndromes also had a shorter disease duration ( 7.8 years) compared to those presenting with pyramidal layer of the CA1 subsector of the hippocampus (stage III; $\mathbf{d}-\mathbf{f}$ ), temporal cortex (stage IV; $\mathbf{g}-\mathbf{i}$ ), frontal cortex (stage $\mathrm{V} ; \mathbf{j}-\mathbf{l}$ ), and visual cortex (stage IV; $\mathbf{m}-\mathbf{o}$ ) are shown. Arrows indicate neurofibrillary tangle that are labeled with both thioflavin-S and PHF-1. Bars $=20 \mu \mathrm{m}$

AD dementia (9.5 years) $(p<0.001)$. Although cognitive decline did not differ, the final MMSE score was lower in cases presenting with atypical clinical syndromes (12 points) compared to those presenting with $\mathrm{AD}$ dementia (15 points) 
Table 1 Demographics and clinicopathologic findings of neuropathologically diagnosed AD cases stratified by clinical diagnostic grouping

Clinical diagnosis of neuropathologically confirmed AD

$p$ value

AD dementia syndrome $\quad$ AD dementia with A typical A typical clinical syndrome clinical syndrome

\begin{tabular}{|c|c|c|c|c|}
\hline \multicolumn{5}{|l|}{ Demographic characteristics } \\
\hline Sample size & $993 / 1356(73 \%)$ & $166 / 1356(12 \%)$ & $197 / 1356(15 \%)$ & \\
\hline Women & $577 / 993(58 \%)$ & $77 / 166(46 \%)$ & $76 / 197(38 \%)$ & $<0.001^{\chi}$ \\
\hline Men & $416 / 993(42 \%)$ & $89 / 166(54 \%)$ & $121 / 197(62 \%)$ & $<0.001^{\chi}$ \\
\hline Education, years & $14(12,16)$ & $14(12,16)$ & $16(12,17)$ & $<0.001$ \\
\hline \multicolumn{5}{|l|}{ Clinical findings } \\
\hline Age onset, years & $73(67,79)$ & $69(62,75)$ & $70(62,76)$ & $<0.001$ \\
\hline Disease duration, years & $9.5(6.6,13)$ & $8.7(7.0,11)$ & $7.8(5.4,10)$ & $<0.001$ \\
\hline Cognitive decline, points/year & $-1.4(-3.4,-0.16)$ & $-1.6(-4.5,-0.22)$ & $-2.8(-5.5,-0.21)$ & 0.311 \\
\hline MMSE final score, points & $15(7,19)$ & $11(4,16)$ & $12(6,19)$ & 0.048 \\
\hline \multicolumn{5}{|l|}{ Neuropathologic findings } \\
\hline Thal amyloid phase & $5(5,5)$ & $5(5,5)$ & $5(5,5)$ & 0.033 \\
\hline Braak tangle stage & $\mathrm{VI}(\mathrm{V}, \mathrm{VI})$ & $\mathrm{VI}(\mathrm{V}, \mathrm{VI})$ & $\mathrm{V}(\mathrm{IV}-\mathrm{V}, \mathrm{VI})$ & $<0.001$ \\
\hline
\end{tabular}

Unless noted, data are presented as median (interquartile range) and assessed in the subset of autopsy-confirmed AD cases from the FLAME cohort regardless of ethnoracial status. Significance tested using Rank Sum Test or ${ }^{{ }}$Chi square test where indicated

$A D$ Alzheimer's disease, MMSE Mini Mental State Examination

$(p=0.048)$. Thal amyloid phase differed $(p=0.033)$, but was less evident where differences diverged as the medians reached Phase five for each group $(p=0.033)$. Braak tangle stage was lower in cases presenting with atypical syndromes (Stage V) compared to those presenting with AD dementia (Stage VI) $(p<0.001)$.

\section{Demographics, clinical findings, and genetic data in neuropathologically-diagnosed Alzheimer's disease cases}

A summary of the demographic, clinical, and genetic findings of autopsy-confirmed AD is found in (Table 2). Within the neuropathologically diagnosed AD cohort, the number of women and men did not significantly differ, with only $n=125(8 \%)$ more women than men identified. A Chi square test was performed with the observed $46 \%$ men and $54 \%$ women against an assumed evenly divided cohort (i.e., $50 \%$ men and 50\% women) did not reveal a significant difference $\left(\chi^{2}=0.180, d f=1, p=0.671\right)$. The lack of difference in frequency between men and women in an autopsy-confirmed AD cohort was consistent when the cases were stratified by ethnoracial status, with only $1(5 \%)$ more man identified in the $n=19$ black Americans and only five (7.5\%) more women identified in the $n=67$ Hispanic Americans. Education was lower in women (13 years for women vs. 16 years for men), whereas age at death (83 vs. 80 years) and age at onset ( 73 vs. 70 years) was greater in women (all $p<0.001$ ). Disease duration was longer in women (10 vs. 8 years), and a greater proportion of women presented clinically with an AD dementia syndrome (79 vs. 66\%) (both $p<0.001)$. Differences were not observed between women and men in frequency of family history of cognitive impairment, cognitive decline measured on longitudinal MMSE, or the final MMSE score. Neither MAPT H1H1 haplotype nor APOE \&4 genotype frequencies differed by sex.

\section{Postmortem findings in neuropathologically diagnosed Alzheimer's disease cases}

Given the significant differences between women and men with respect to age at onset, disease duration, and education, these variables were incorporated into regression models to account for the contribution of the observed clinical differences (Table 3). AD brains were smaller for women than men (980 vs. 1120 grams), and women had higher Braak tangle stage (VI vs. V-VI) (both adjusted $p<0.001)$. The Thal amyloid phase approached significance, with women having slightly more amyloid (adj. $p=0.055$ ). Lewy body disease, especially diffuse Lewy body disease, was higher in men (overall $27 \%$ in men and $21 \%$ in women), but this did not survive adjustment. As expected [24], men were overrepresented in hippocampal sparing AD (14 vs. 7\%) and women were more than twice as frequent in limbic predominant AD (18 vs. 9\%) (adj. $p=0.052)$. A higher frequency of TDP-43 positivity was found in women (44 vs. 35\%), but this difference did not survive after adjusting for clinical variables. The 
Table 2 Demographics, clinical findings, and genetic data in neuropathologically diagnosed Alzheimer's disease cases from the FLAME study

\begin{tabular}{|c|c|c|c|}
\hline & Men & Women & $p$ value \\
\hline \multicolumn{4}{|l|}{ Demographic characteristics } \\
\hline Sample size & $750 / 1625(46 \%)$ & $875 / 1625(54 \%)$ & \\
\hline Ethnoracial composition $^{\mathrm{a}}$ & & & $0.850^{x}$ \\
\hline Black American & $10 / 750(1 \%)$ & $9 / 875(1 \%)$ & \\
\hline Hispanic American & $31 / 750(4 \%)$ & $36 / 875(4 \%)$ & \\
\hline White American & $709 / 750(94 \%)$ & $830 / 875(95 \%)$ & \\
\hline Family history, $\%$ positive & $236 / 735(32 \%)$ & $279 / 841(33 \%)$ & $0.692^{\chi}$ \\
\hline Education & $16(12,16)$ & $13(12,16)$ & $<0.001$ \\
\hline Age at death, years & $80(73,84)$ & $83(78,88)$ & $<0.001$ \\
\hline \multicolumn{4}{|l|}{ Clinical findings } \\
\hline Age onset, years & $70(64,76)$ & $73(66,79)$ & $<0.001$ \\
\hline Disease duration, years & $8(6,11)$ & $10(7,13)$ & $<0.001$ \\
\hline Cognitive decline, pts/year ${ }^{\mathrm{b}}$ & $-1(-4,-0.2)$ & $-1(-4,-0.2)$ & 0.672 \\
\hline MMSE final score, points ${ }^{c}$ & $13(6,19)$ & $12(6,19)$ & 0.860 \\
\hline Clinical diagnosis, $\%$ & & & $<0.001^{\chi}$ \\
\hline AD dementia syndrome & $416 / 626(66 \%)$ & $577 / 730(79 \%)$ & \\
\hline AD with a typical clinical syndrome & $89 / 626(14 \%)$ & $77 / 730(11 \%)$ & \\
\hline Atypical clinical syndrome & $121 / 626(20 \%)$ & $76 / 730(10 \%)$ & \\
\hline \multicolumn{4}{|l|}{ Genetic findings } \\
\hline MAPT H1H1, \%positive & $246 / 425(58 \%)$ & $312 / 508(61 \%)$ & $0.303^{x}$ \\
\hline$A P O E \& 4, \%$ positive & $335 / 550(61 \%)$ & $412 / 627(66 \%)$ & $0.100^{x}$ \\
\hline
\end{tabular}

$A D$ Alzheimer's disease, MMSE Mini Mental State Examination, MAPT Microtubule associated protein Tau, APOE Apolipoprotein $\mathrm{E}$

${ }^{a}$ Ethnoracial group is self-reported

${ }^{\mathrm{b}}$ Cognitive decline was measured in 130 women and 143 men who had three or more MMSE scores available for analysis

${ }^{c}$ Last MMSE score was tested within 3 years of death (time from test date to death did not differ) and available for 122 women and 181 men. Unless noted, data are presented as median (interquartile range) and assessed in the subset of autopsy-confirmed AD cases from the FLAME cohort regardless of ethnoracial status. Significance tested using Rank Sum Test or ${ }^{\chi}$ Chi square test where indicated frequency of co-existing hippocampal sclerosis did not differ between women and men. Cerebrovascular disease was more common in women ( 29 vs. $22 \%$ ), but did not survive after adjustment.

We examined the frequency of neuropathologic diagnosis of AD stratified by decade of age at death, ranging from the 6 th (i.e., 50-59) to the 11 th decade (i.e., 100-109) [suppl. Table 1 (Online Resource 3)]. Prior to the 9th decade of life, men with AD were overrepresented, with a peak frequency in the 7 th decade of life. From the 9th decade of life onward, women with AD were overrepresented, with an observed exponential increase. No men with $\mathrm{AD}$ were observed in the centenarian cohort. Figure 3 illustrates frequency plots with an overlay of clinical presentation of AD dementia. Coinciding with an overrepresentation of women in the 9th decade, clinical presentation of AD dementia nearly doubled compared to that observed in the 6 th decade of life.

\section{Neuropathologic distribution of amyloid- $\beta$ plaque and neurofibrillary tangle by age and sex}

Cortical and limbic amyloid- $\beta$ plaque counts per $3 \mathrm{~mm}^{2}(10 \mathrm{x}$ magnification) (Fig. 4) and neurofibrillary tangle counts per $0.125 \mathrm{~mm}^{2}$ (40× magnification) (Fig. 5) in women and men were separately examined by terminal decade. Amyloid- $\beta$ plaques were found to steadily decrease with advancing age across association cortices, primary cortices, and hippocampal regions. This pattern observed was similarly observed in men and women [suppl. Table 2 (Online Resource 3), Fig. 4]. Regional distribution of neurofibrillary tangle counts in the hippocampus and neocortex of men showed an overall pattern of a steady decrease in severity with increasing age [suppl. Table 3 (Online Resource 3), Fig. 5]. Neurofibrillary tangle counts in men remained higher in the neocortex during the 6 th and 7 th decades, with more than a twofold decrease observed in the 8 th decade onwards. In contrast, 
Table 3 Postmortem findings in neuropathologically diagnosed Alzheimer's disease cases from the FLAME study

\begin{tabular}{|c|c|c|c|c|c|c|}
\hline \multirow[t]{2}{*}{ Multiple linear } & \multirow[t]{2}{*}{ Men } & \multirow[t]{2}{*}{ Women } & \multicolumn{2}{|l|}{ Unadjusted model } & \multicolumn{2}{|l|}{ Adjusted model } \\
\hline & & & Coefficient (SE) & $p$ value & Coefficient (SE) & $p$ value \\
\hline Brain weight $(\mathrm{g})$ & $1120(1020,1210)$ & $980(900,1060)$ & $141(7.2)$ & $<0.001$ & $131(8.8)$ & $<0.001$ \\
\hline Thal amyloid phase & $5(5,5)$ & $5(5,5)$ & $-0.065(0.032)$ & 0.039 & $-0.085(0.044)$ & 0.055 \\
\hline Braak tangle stage & V-VI (V,VI) & VI $(\mathrm{V}, \mathrm{VI})$ & $-0.16(0.033)$ & $<0.001$ & $-0.19(0.044)$ & $<0.001$ \\
\hline Lewy body disease & & & $0.16(0.056)$ & 0.005 & $0.13(0.080)$ & 0.104 \\
\hline None & $541 / 736(73 \%)$ & 686/866 (79\%) & & & & \\
\hline Brainstem & $7 / 736(1 \%)$ & $7 / 866(1 \%)$ & & & & \\
\hline Transitional & $70 / 736(10 \%)$ & $72 / 866(8 \%)$ & & & & \\
\hline Diffuse & $118 / 736(16 \%)$ & $101 / 866(12 \%)$ & & & & \\
\hline AD subtype & & & $-0.16(0.026)$ & $<0.001$ & $0.069(0.035)$ & 0.052 \\
\hline Hippocampal sparing & $94 / 653(14 \%)$ & $53 / 747(7 \%)$ & & & & \\
\hline Typical & $498 / 653(76 \%)$ & $562 / 747(75 \%)$ & & & & \\
\hline Limbic & $61 / 653(9 \%)$ & $132 / 747(18 \%)$ & & & & \\
\hline Multiple logistic & & & Odds ratio $(5 \%, 95 \%)$ & $p$ value & Odds ratio $(5 \%, 95 \%)$ & $p$ value \\
\hline TDP-43 positive & $151 / 428(35 \%)$ & $226 / 511(44 \%)$ & $0.69(0.53,0.90)$ & 0.005 & $1.0(0.70,1.4)$ & 0.968 \\
\hline Hippocampal sclerosis ${ }^{\mathrm{a}}$ & $49 / 750(6 \%)$ & $73 / 875(8 \%)$ & $0.77(0.53,1.12)$ & 0.169 & $1.0(0.63,1.7)$ & 0.855 \\
\hline Cerebrovascular disease & $167 / 750(22 \%)$ & 253/875 (29\%) & $0.70(0.56,0.88)$ & 0.002 & $1.0(1.0,1.0)$ & 0.830 \\
\hline
\end{tabular}

$g$ Grams, $A D$ Alzheimer's disease, TDP-43 Tar DNA binding protein-43

${ }^{a}$ All hippocampal sclerosis cases are TDP-43 positive. Unless noted, data are presented as median (interquartile range) and assessed in the subset of autopsy-confirmed AD cases from the FLAME cohort regardless of ethnoracial status. Multiple linear and multiple logistic regression modeling was used to adjust for the potential contribution of significant clinical parameters (age onset, disease duration, education), where neuropathologic variable was the dependent variable and sex $($ Female $=0$, Male $=1)$ was input as an independent variable

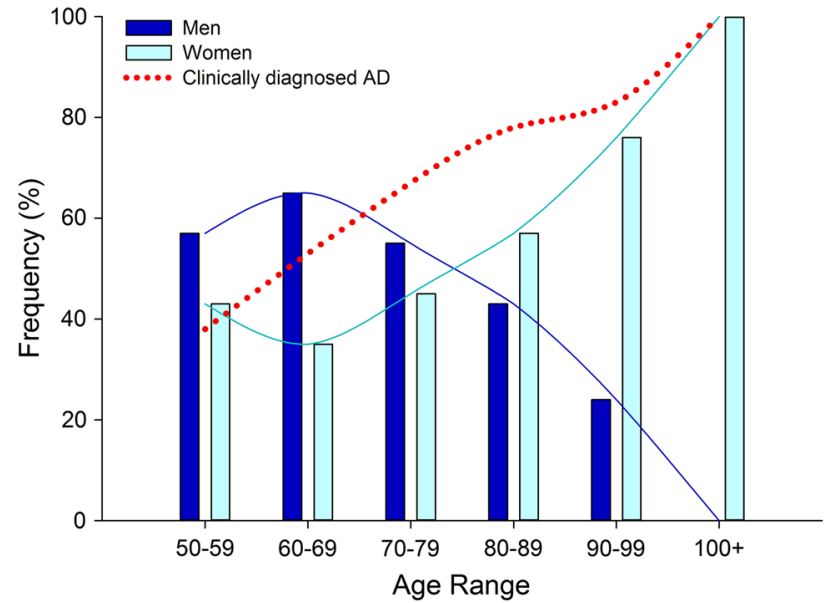

Fig. 3 Disproportionate frequency of women and men neuropathologically diagnosed as Alzheimer's disease across six decades. Frequency plots of age at death revealed an inverted U-shaped curve in men with autopsy-confirmed $\mathrm{AD}$, demonstrating a higher frequency of death in their 7 th decade. In comparison, age at death in women with autopsy-cofirmed $\mathrm{AD}$ was found to be overrepresented in later decades-particularly the 10th and 11th decades of life. The frequency of autopsied $\mathrm{AD}$ cases that presented clinically with an $\mathrm{AD}$ dementia without a non-AD or atypical clinical syndrome in the differential demonstrated a strong age-associated increase in the accuracy of the diagnosis neurofibrillary tangles increased steadily in hippocampal subregions of women with increasing age. Similar to men, neurofibrillary tangle severity decreased in the neocortex with increasing age, with a notable clustering in the 6th and 7 th decade. A twofold decline was observed for women in the 9th decade onwards. The ratio between neurofibrillary tangles in women and neurofibrillary tangles in men within each brain region was stratified by age at death (Table 4). Women were more severely affected in the hippocampalby-age matrix [9/10 (90\%)]. This observation lessened in cortical regions as women were more severely affected in a little more than half of the association cortex-by-age matrix [9/15 (60\%)] and less than half of the primary cortex-byage matrix [4/10 (40\%)]. In order to validate these findings, we examined age-associated sex differences in neuropathologic severity using immunohistochemistry in a subset of neuropathologically diagnosed Alzheimer's disease cases. Thioflavin-S strongly correlated with PHF-1 immunopositive neurofibrillary tangle pathology in the CA1 $(R=0.774$, $p<0.001)$, subiculum $(R=0.715, p<0.001)$, superior temporal $(R=0.546, p<0.001)$, inferior parietal $(R=0.785$, $p<0.001)$, and middle frontal $(R=0.823, p<0.001)$. A similar pattern of PHF-1 involvement was observed across neuroanatomic regions when examined by terminal decade in men and women [suppl. Table 4 (Online Resource 6)]. 
Fig. 4 Cortical and hippocampal amyloid- $\beta$ plaques quantitatively differ by age at death. Stacked bar charts graphically display the neuroanatomic distribution of amyloid- $\beta$ plaque counts per $3 \mathrm{~mm}^{2}$. The colored cells represent the median value of amyloid- $\beta$ plaque counts (x-axis) per given region (color coded), age at death (y-axis), and sex (right vs. left). The total width by age group is an additive sum of amyloid- $\beta$ plaques, which demonstrates lessening pathology with advancing age. (Left) Men and (Right) women shared a similar pattern with a ceiling effect noted in the association cortices (frontal, temporal, and parietal). Both primary cortices (visual and motor) and hippocampal subregions (subiculum and CA1) were found to decrease as age increases

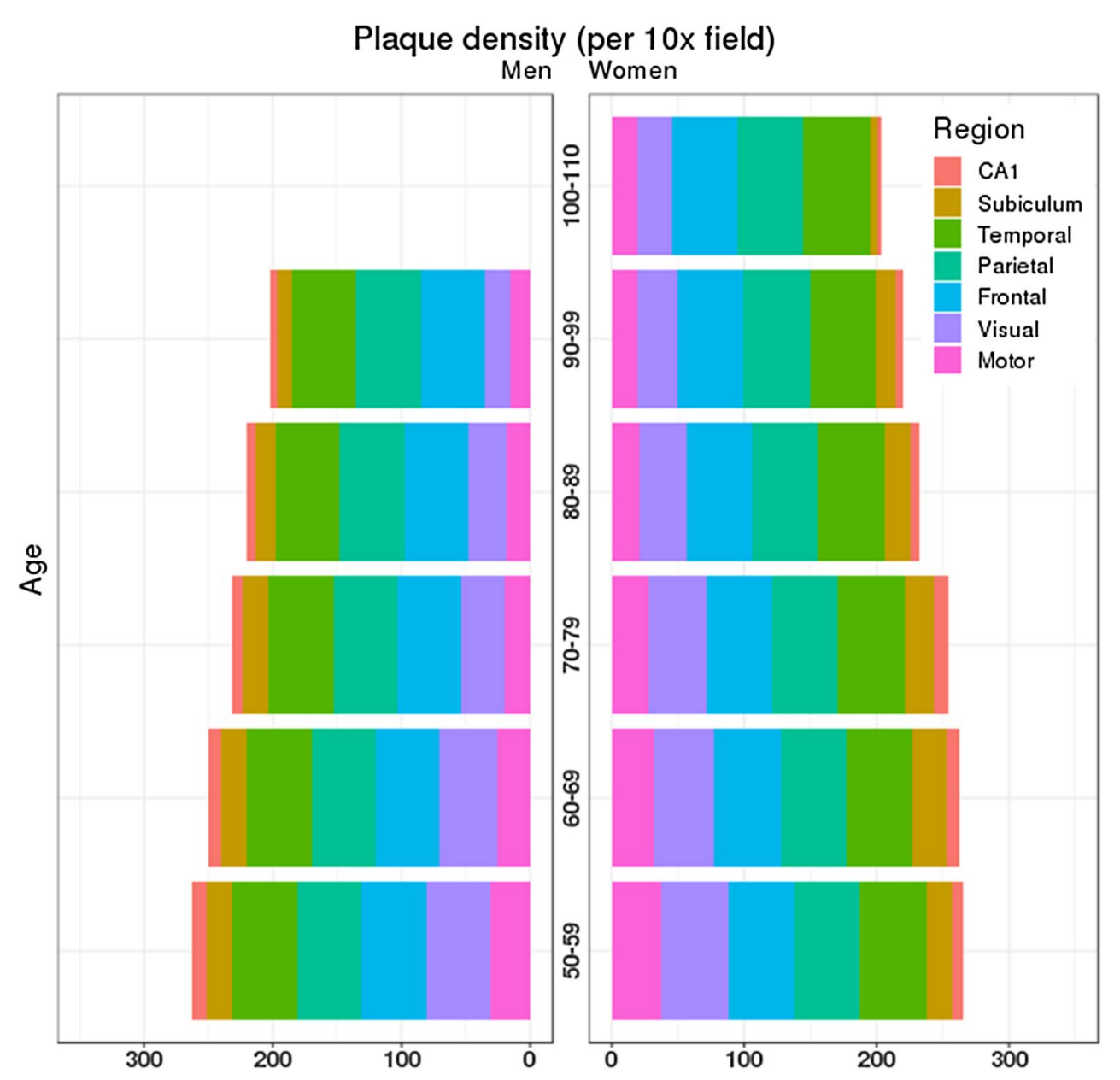

\section{Discussion}

In the FLAME cohort, the overall frequency of autopsyconfirmed AD did not differ between women and men; however, men less often had an amnestic, multidomain clinical syndrome typical of AD dementia. As expected based upon societal gender-bias, women had lower educational levels. Women also had lower brain weights, older age at onset, longer disease duration, and older age at death. Severity of amyloid- $\beta$ plaque pathology and neurofibrillary tangle pathology was greater in women compared to men. Interestingly, the frequency of autopsy-confirmed $\mathrm{AD}$ was highest among men in their $60 \mathrm{~s}$, while women had an exponential increase from their $60 \mathrm{~s}$ into their late $90 \mathrm{~s}$.

Our study investigating a large autopsy-confirmed AD cohort did not find an overrepresentation of women, but did find that men were less likely than women to present clinically with an $\mathrm{AD}$ dementia syndrome. A number of previous clinical studies, however, have found women are at a higher risk of AD than men [3,20]. Others have shown that higher risk is more specific to older women $[10,15,28$, 31 ], supporting our observation of overrepresentation of women in their $80 \mathrm{~s}$ onward in an autopsy-confirmed AD cohort. Still others found no difference in incidence risk of $\mathrm{AD}$ in women, or once they controlled for age the risk was mitigated [19, 28, 29]. Collectively, these studies support our finding of overall similar frequency of $\mathrm{AD}$ in women and men.

Our study is one of the first to highlight over representation of autopsy-confirmed AD in men in the 7th decade (i.e., 60-69). Men were more likely to present clinically with a non-amnestic or atypical clinical syndrome (e.g., corticobasal syndrome, frontotemporal dementia) and to have a younger age at onset. Diagnostic accuracy of autopsyconfirmed $\mathrm{AD}$ was lower in men than women in this study, especially in the 6th and 7th decades, when men more commonly came to autopsy. Men were also found to have greater neocortical neurofibrillary tangle pathology, relative to hippocampal tangles compared to women. In contrast, women were overrepresented in later decades and had greater hippocampal neurofibrillary tangle pathology relative to neocortex. The above mentioned findings, which are also mirrored in tau PET biomarker studies [26], may be in concordance with the observation in epidemiological studies that mild cognitive impairment is more frequently diagnosed among men than in women [27]. One interpretation may be 
Fig. 5 Cortical and hippocampal neurofibrillary tangles quantitatively differ by age at death and sex. Stacked bar charts graphically display the neuroanatomic distribution of neurofibrillary tangle counts per $0.125 \mathrm{~mm}^{2}$. The colored cells represent the median value of neurofibrillary tangle counts (x-axis) per given region (color coded), age at death (y-axis), and sex (right vs. left). The total width by age group is an additive sum of neurofibrillary tangles, which demonstrates lessening pathology with advancing age. (Left) Men and (Right) women differed in the age-associated involvement of the hippocampus, with women showing a more pronounced increase in their neurofibrillary tangle counts with advancing age. Both association and primary cortices were observed to have fewer tangles with each progressive decade in women and men. A shift between the 6 th and 7 th decades to the 8th decade onward was more pronounced in men, whereas the shift to fewer cortical tangles in women occurred more so in the 9 th decade onward

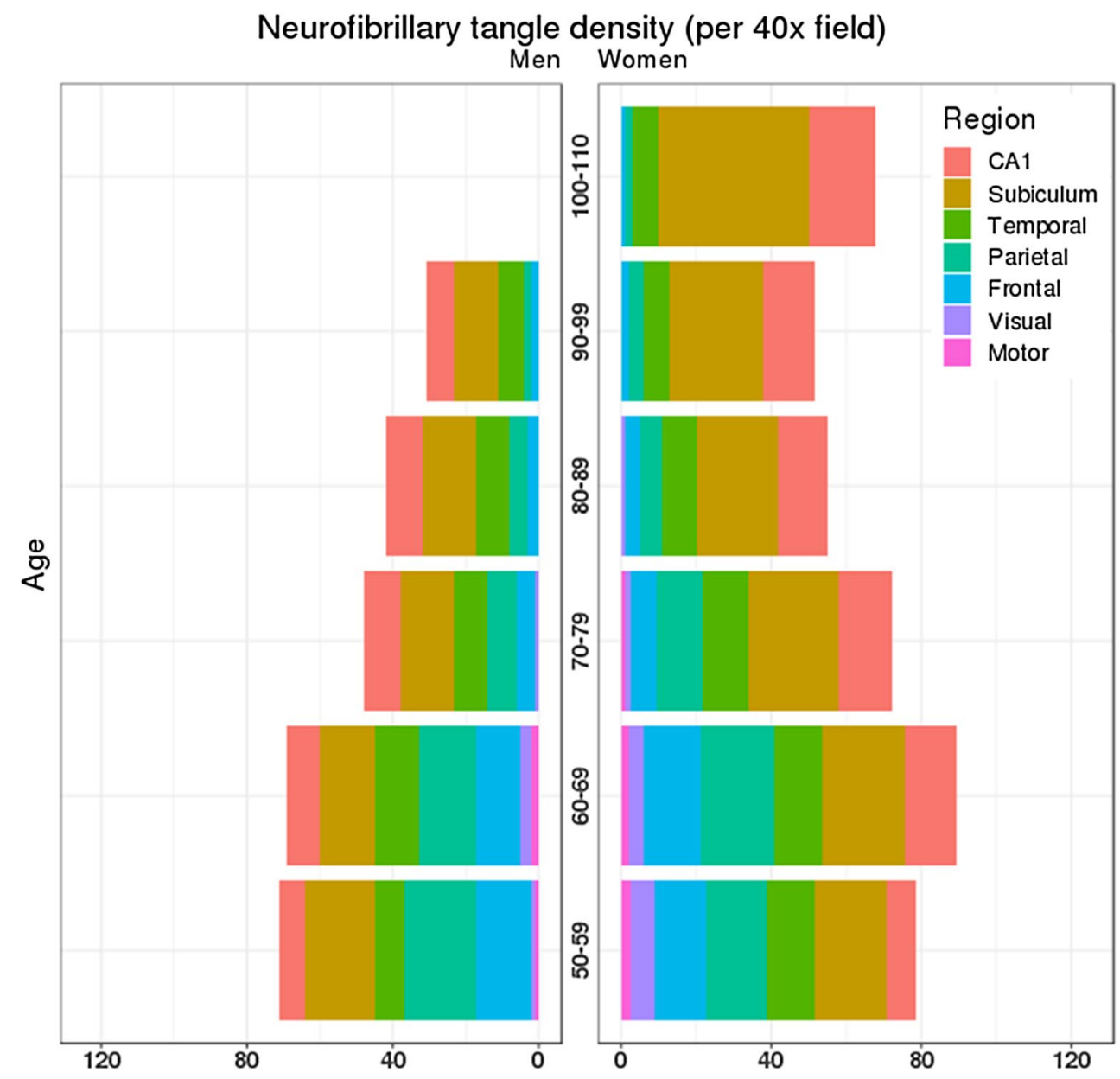

Table 4 Ratio comparison of regionally distributed neurofibrillary tangles by age range between men and women in neuropathologically diagnosed Alzheimer's disease

\begin{tabular}{|c|c|c|c|c|c|c|c|}
\hline \multirow[b]{2}{*}{ Age at death } & \multicolumn{2}{|c|}{ Hippocampal } & \multicolumn{3}{|c|}{ Association cortices } & \multicolumn{2}{|c|}{ Primary cortices } \\
\hline & CA1 & Subiculum & Temporal & Parietal & Frontal & Visual & Motor \\
\hline $50-59$ years & 1.1 & 1.0 & 1.5 & 0.8 & 0.9 & 6.0 & 2.0 \\
\hline $60-69$ years & 1.6 & 1.5 & 1.0 & 1.3 & 1.3 & 1.3 & 1.0 \\
\hline $70-79$ years & 1.4 & 1.6 & 1.4 & 1.5 & 1.6 & 2.0 & - \\
\hline $80-89$ years & 1.3 & 1.5 & 1.0 & 1.2 & 1.3 & - & - \\
\hline 90-99 years & 1.8 & 2.1 & 1.0 & 2.0 & 1.0 & - & - \\
\hline
\end{tabular}

The median number of tangles in women was divided by the median number in men. Values $<1$ (green scale) indicate greater severity in women, whereas values $>1$ (yellow scale) indicate greater severity in men. A value $=1$ indicates the same median value was identified in both men and women. Note: A dash (-) symbol indicates that the median tangle density was equal to 0 for both in men and women, with the exception of Visual tangles $=1$ for women and $=0$ for men

that women who have more severe memory impairment may abruptly transition from normal to a more severely impaired cognitive state, whereas men with atypical clinical syndromes may have a slower transition from a mild cognitive impairment state to dementia.

We and others have shown that the rate of cognitive decline does not differ between women and men, but that women are more likely to have overt dementia $[5,6]$. Our data further demonstrate that neurofibrillary tangle counts in the hippocampus, across all ages, are more severe among women than men and that limbic predominant AD subtype is more common among women. Taken together with findings from neuroimaging studies demonstrating hippocampal atrophy occurs at a faster rate in women [2], our study supports the observation that women are more likely than men to present with a typical amnestic dementia. We hypothesize that women in later decades have a high frequency of correct diagnosis as $\mathrm{AD}$ dementia because $\mathrm{AD}$ neuropathology is 
more frequent in women and they have greater hippocampal involvement relative to the neocortex, leading to an amnestic syndrome.

In this study we extend our current knowledge by examining quantitative neurofibrillary tangle counts measures in association cortices, primary cortices, and hippocampal subsectors. Our findings suggest that there is an interaction between age and sex, resulting in selective neuroanatomic susceptibility to neurofibrillary pathology in different decades of life. Importantly, the neocortex may be especially vulnerable to neurofibrillary pathology in younger onset individuals, especially men, and to have a more rapid progression. Conversely, older aged individuals were found to have significantly reduced cortical pathology [8]. Women with autopsy-confirmed AD have been consistently found to be older at death $[4,5,13,32]$, but our study additionally suggests that men may be younger at onset of cognitive symptoms and have a shorter disease duration.

Our primary goal was to investigate the hypothesis that epidemiological studies showing greater prevalence of $\mathrm{AD}$ dementia in women may be biased by the sex- and ageinfluenced distribution of Alzheimer type pathology, which results in a disproportionate number of men with atypical clinical presentations. Our results support this hypothesis and point to differences in selective vulnerability to neurofibrillary tangle pathology as a plausible biological factor underlying differences in frequency of clinical diagnoses. Other biological factors that may have a bearing on our findings include the impact of sex hormones on neuronal vulnerability [30], as well as developmental differences in brain reserve [23, 33, 35]. One of the most important considerations when investigating sex differences in an aging cohort is differential survival of men and women, relating to their cardiovascular risk profile [10]. Thus, survival differences, resulting from differing cardiac risk profiles, could contribute to the observed lower frequency of $\mathrm{AD}$ in men in their later decades, as well as a greater frequency of men diagnosed with mild cognitive impairment.

This study has several limitations inherent to autopsybased studies derived from specialty clinics or from self-referral. Although data were provided on clinical progression, the FLAME cohort is a cross-sectional and retrospective study. We did not observe overall sex differences in women and men, but there may be inherent bias among individuals who elected to participate in autopsy program [21]. Interestingly, we found that the overall FLAME cohort was comprised of $51 \%$ men and $49 \%$ women, suggesting brain donations from specialty clinics or from self-referral may not be sex-biased. Another potential limitation is that patients or the caregivers of patients with atypical clinical syndromes may be more likely to seek autopsy confirmation. Thus, our clinical findings may not be generalizable to population or epidemiologic-based cohorts. It should also be noted that our reported findings on cognitive decline were derived from a much smaller cohort with three or more available MMSE scores used to calculate rate of change. Thioflavin-S is a fluorescent dye that binds to $\beta$-pleated sheets and thus readily binds to both amyloid- $\beta$ plaques and neurofibrillary tangles. Although immunohistochemical methods were used to validate thioflavin-S findings, it should be noted that differences in sensitivity may occur when assessing presence of pathology. An unbiased stereology approach was not employed to quantify neurofibrillary tangle and senile plaque data. To offset this limitation, neuropathologic lesions were quantified as counts per microscopic field across a large series of autopsy-confirmed AD cases. We did not observe sex-based frequency differences within black Americans or Hispanic Americans, but further examination of these underserved cohorts should be performed to identify whether our findings remain consistent in other diverse cohorts of autopsy-confirmed AD. Last, information on cause of death is rarely available, thus limiting further investigation into survival bias.

From a historical perspective, sex differences in research studies have not been adequately investigated and in some circumstances women have even been excluded from participating in many studies. This bias against inclusion of women ultimately prompted a federal mandate in the United States requiring inclusion of women in research studies [11]. Sex is often used as a covariate with balanced cohorts being investigated, but by statistically adjusting for sex, important biological clues underlying disease mechanisms may be neglected. Our data derived from autopsy-confirmed AD did not reveal a sex bias in terms of recruitment and consent for autopsy and they demonstrate that women and men have a similar frequency of AD. Sex-based differences in the age at onset, selective vulnerability of neocortical and limbic brain regions and the resulting clinical presentations may contribute to previous observations in clinical studies of AD being more common in women. These present observations should not take away from progress being made toward women's health initiatives [12], but instead should be used to emphasize the importance of considering neuropathologic and biological differences in young-onset $\mathrm{AD}$ (i.e., $<65$ years) compared to $\mathrm{AD}$ in the oldest-old (i.e., $>85$ years). The bearing of sex and age, as well as differential rates of progression of memory impairment, may have important implications for therapeutic trials, which may benefit from stratification of women and men, and/or by age at onset when assessing efficacy.

Acknowledgements Mrs. Liesinger, Dr. Duara, Dr. Aziz, Mrs. Hinkle, Ms. DiLello, Ms. Johnson, Dr. Ross, Dr. Ertekin-Taner, and Dr. Dickson have nothing to disclose. Dr. Graff-Radford reports grants from Biogen, grants from Lilly, grants from Novartis, grants from Axovant, grants from Janssen during the conduct of the study. Dr. Murray reports grants from Florida Department of Health, Ed and Ethel Moore 
Alzheimer's Disease Research Program (6AZ01, 8AZ06), grants from National Institute on Aging (R01 AG054449), grants from Gerstner Family Career Development Award during the conduct of the study; personal fees from Avid Radiopharmaceuticals, Inc., outside the submitted work. We thank the patients and their families for their generous brain donations to help further our knowledge of Alzheimer's disease.

Open Access This article is distributed under the terms of the Creative Commons Attribution 4.0 International License (http://creativeco mmons.org/licenses/by/4.0/), which permits unrestricted use, distribution, and reproduction in any medium, provided you give appropriate credit to the original author(s) and the source, provide a link to the Creative Commons license, and indicate if changes were made.

\section{References}

1. Amador-Ortiz C, Lin WL, Ahmed Z, Personett D, Davies P, Duara $R$ et al (2007) TDP-43 immunoreactivity in hippocampal sclerosis and Alzheimer's disease. Ann Neurol 61:435-445. https://doi. org/10.1002/ana.21154

2. Ardekani BA, Convit A, Bachman AH (2016) Analysis of the MIRIAD data shows sex differences in hippocampal atrophy progression. J Alzheimers Dis 50:847-857. https://doi.org/10.3233/ jad- 150780

3. Bachman DL, Wolf PA, Linn RT, Knoefel JE, Cobb JL, Belanger AJ et al (1993) Incidence of dementia and probable Alzheimer's disease in a general population: the Framingham study. Neurology 43:515. https://doi.org/10.1212/wnl.43.3_part_1.515

4. Barker WW, Luis CA, Kashuba A, Luis M, Harwood DG, Loewenstein D et al (2002) Relative frequencies of Alzheimer disease, Lewy body, vascular and frontotemporal dementia, and hippocampal sclerosis in the state of Florida brain bank. Alzheimer Dis Assoc Disord 16:203-212

5. Barnes LL, Wilson RS, Bienias JL, Schneider JA, Evans DA, Bennett DA (2005) Sex differences in the clinical manifestations of Alzheimer disease pathology. Arch Gen Psychiatry 62:685-691. https://doi.org/10.1001/archpsyc.62.6.685

6. Barnes LL, Wilson RS, Schneider JA, Bienias JL, Evans DA, Bennett DA (2003) Gender, cognitive decline, and risk of AD in older persons. Neurology 60:1777-1781. https://doi.org/10.1212/01. wnl.0000065892.67099.2a

7. Beach TG, White CL, Hamilton RL, Duda JE, Iwatsubo T, Dickson DW et al (2008) Evaluation of alpha-synuclein immunohistochemical methods used by invited experts. Acta Neuropathol 116:277-288. https://doi.org/10.1007/s00401-008-0409-8

8. Berg L, McKeel DW, Miller JP, Storandt M, Rubin EH, Morris JC et al (1998) Clinicopathologic studies in cognitively healthy aging and Alzheimer disease-relation of histologic markers to dementia severity, age, sex, and apolipoprotein E genotype. Arch Neurol 55:326-335. https://doi.org/10.1001/archneur.55.3.326

9. Braak H, Braak E (1991) Neuropathological stageing of Alzheimer-related changes. Acta Neuropathol 82:239-259

10. Chêne G, Beiser A, Au R, Preis SR, Wolf PA, Dufouil C et al (2015) Gender and incidence of dementia in the Framingham heart study from mid-adult life. Alzheimer's Dementia 11:310 320. https://doi.org/10.1016/j.jalz.2013.10.005

11. Clayton JA, Collins FS (2014) Policy: NIH to balance sex in cell and animal studies. Nature 509:282-283

12. Craig MC, Maki PM, Murphy DG (2005) The women's health initiative memory study: findings and implications for treatment. Lancet Neurol 4:190-194. https://doi.org/10.1016/s1474 $-4422(05) 01016-1$
13. Filon JR, Intorcia AJ, Sue LI, Vazquez Arreola E, Wilson J, Davis $\mathrm{KJ}$ et al (2016) Gender differences in Alzheimer disease: brain atrophy, histopathology burden, and cognition. J Neuropathol Exp Neurol 75:748-754. https://doi.org/10.1093/jnen/nlw047

14. Galton CJ, Patterson K, Xuereb JH, Hodges JR (2000) Atypical and typical presentations of Alzheimer's disease: a clinical, neuropsychological, neuroimaging and pathological study of 13 cases. Brain 123(Pt 3):484-498

15. Hebert LE, Scherr PA, McCann JJ, Beckett LA, Evans DA (2001) Is the risk of developing Alzheimer's disease greater for women than for men? Am J Epidemiol 153:132-136

16. Jellinger KA, Attems J (2003) Incidence of cerebrovascular lesions in Alzheimer's disease: a postmortem study. Acta Neuropathol 105:14-17. https://doi.org/10.1007/s00401-002-0634-5

17. Khachaturian ZS (1985) Diagnosis of Alzheimer's disease. Arch Neurol 42:1097-1105

18. Koga S, Ono M, Sahara N, Higuchi M, Dickson DW (2017) Fluorescence and autoradiographic evaluation of tau PET ligand PBB3 to alpha-synuclein pathology. Mov Disord 32:884-892. https:// doi.org/10.1002/mds.27013

19. Kukull WA, Higdon R, Bowen JD et al (2002) Dementia and alzheimer disease incidence: a prospective cohort study. Arch Neurol 59:1737-1746. https://doi.org/10.1001/archneur.59.11.1737

20. Launer LJ, Andersen K, Dewey ME, Letenneur L, Ott A, Amaducci LA et al (1999) Rates and risk factors for dementia and Alzheimer's disease: results from EURODEM pooled analyses. EURODEM incidence research group and work groups. European studies of Dementia. Neurology 52:78-84

21. Maraganore DM, Anderson DW, Bower JH, McDonnell SK, Rocca WA (1999) Autopsy patterns for Parkinson's disease and related disorders in Olmsted county, Minnesota. Neurology 53:1342-1344

22. McKeith IG, Boeve BF, Dickson DW, Halliday G, Taylor JP, Weintraub D et al (2017) Diagnosis and management of dementia with Lewy bodies: fourth consensus report of the DLB consortium. Neurology 89:88-100. https://doi.org/10.1212/wnl.00000 00000004058

23. Mielke MM, Vemuri P, Rocca WA (2014) Clinical epidemiology of Alzheimer's disease: assessing sex and gender differences. Clin Epidemiol 6:37-48. https://doi.org/10.2147/clep.s37929

24. Murray ME, Graff-Radford NR, Ross OA, Petersen RC, Duara R, Dickson DW (2011) Neuropathologically defined subtypes of Alzheimer's disease with distinct clinical characteristics: a retrospective study. Lancet Neurol 10:785-796. https://doi.org/10.1016/ s1474-4422(11)70156-9

25. Murray ME, Lowe VJ, Graff-Radford NR, Liesinger AM, Cannon A, Przybelski SA et al (2015) Clinicopathologic and 11C-Pittsburgh compound B implications of Thal amyloid phase across the Alzheimer's disease spectrum. Brain 138:1370-1381. https://doi. org/10.1093/brain/awv050

26. Ossenkoppele R, Schonhaut DR, Scholl M, Lockhart SN, Ayakta N, Baker SL et al (2016) Tau PET patterns mirror clinical and neuroanatomical variability in Alzheimer's disease. Brain 139:1551-1567. https://doi.org/10.1093/brain/aww027

27. Petersen RC, Roberts RO, Knopman DS, Geda YE, Cha RH, Pankratz VS et al (2010) Prevalence of mild cognitive impairment is higher in men. the mayo clinic study of aging. Neurology 75:889-897. https://doi.org/10.1212/wnl.0b013e3181f11d85

28. Plassman BL, Langa KM, Fisher GG, Heeringa SG, Weir DR, Ofstedal MB et al (2007) Prevalence of dementia in the United States: the aging, demographics, and memory study. Neuroepidemiology 29:125-132. https://doi.org/10.1159/000109998

29. Rocca WA, Cha RH, Waring SC, Kokmen E (1998) Incidence of dementia and Alzheimer's disease: a reanalysis of data from Rochester, Minnesota, 1975-1984. Am J Epidemiol 148:51-62 
30. Rosario ER, Chang L, Head EH, Stanczyk FZ, Pike CJ (2011) Brain levels of sex steroid hormones in men and women during normal aging and in Alzheimer's disease. Neurobiol Aging 32:604-613. https://doi.org/10.1016/j.neurobiola ging.2009.04.008

31. Ruitenberg A, Ott A, van Swieten JC, Hofman A, Breteler MMB (2001) Incidence of dementia: does gender make a difference? Neurobiol Aging 22:575-580. https://doi.org/10.1016/S0197 $-4580(01) 00231-7$

32. Savva GM, Wharton SB, Ince PG, Forster G, Matthews FE, Brayne C (2009) Age, neuropathology, and dementia. N Engl J Med 360:2302-2309. https://doi.org/10.1056/nejmoa0806142

33. Smith CD, Chebrolu H, Wekstein DR, Schmitt FA, Markesbery WR (2007) Age and gender effects on human brain anatomy: a voxel-based morphometric study in healthy elderly. Neurobiol Aging 28:1075-1087. https://doi.org/10.1016/j.neurobiola ging.2006.05.018

34. Snowden JS, Stopford CL, Julien CL, Thompson JC, Davidson Y, Gibbons L et al (2007) Cognitive phenotypes in Alzheimer's disease and genetic risk. Cortex 43:835-845
35. Stern Y (2012) Cognitive reserve in ageing and Alzheimer's disease. Lancet Neurol 11:1006-1012. https://doi.org/10.1016/s1474 $-4422(12) 70191-6$

36. Thal DR, Rub U, Orantes M, Braak H (2002) Phases of A betadeposition in the human brain and its relevance for the development of AD. Neurology 58:1791-1800

37. Vina J, Lloret A (2010) Why women have more Alzheimer's disease than men: gender and mitochondrial toxicity of amyloid-beta peptide. J Alzheimers Dis 20(Suppl 2):S527-S533. https://doi. org/10.3233/jad-2010-100501

38. Winblad B, Amouyel P, Andrieu S, Ballard C, Brayne C, Brodaty $\mathrm{H}$ et al (2016) Defeating Alzheimer's disease and other dementias: a priority for European science and society. Lancet Neurol 15:455-532. https://doi.org/10.1016/s1474-4422(16)00062-4

39. Zhang YJ, Xu YF, Cook C, Gendron TF, Roettges P, Link CD et al (2009) Aberrant cleavage of TDP-43 enhances aggregation and cellular toxicity. Proc Natl Acad Sci USA 106:7607-7612. https ://doi.org/10.1073/pnas.0900688106 